1 Hacettepe Journal of Mathematics and Statistics

$\bigcap$ Volume 46 (1) (2017), 9-14

\title{
Generalizations of metrics and partial metrics
}

Over half a century ago, Dr. L. M. Brown made an important discovery that did not become a theorem in any paper: You can work and live happily and productively with people of different cultures, and the result will be good for all. Dr. Brown passed on this message through the example that participants saw in the topology conferences he organized. The two authors met at one of these in summer 2001 and talked about future collaboration. We officially began working in October 2002 along with Steve Matthews in England for a year. Since then we have worked on developing and studying generalized metrics, among other topics. So our special thanks go to Dr. Brown for organizing these Hacetteppe conferences. Due to him our lives and research are much more enjoyable and productive than they would be otherwise.

$$
\text { Ralph Kopperman * and Homeira Pajoohesh }{ }^{\dagger}
$$

\begin{abstract}
In [14] $k$-metric spaces were defined for certain $\ell$-group applications, by weakening the metric triangle inequality. In this article we show that much of the theory of metric spaces, including the Banach fixed point theorem extends to these spaces.
\end{abstract}

Keywords: partial metrics, variety of $\ell$-groups, $k$-metrics

2000 AMS Classification: 06F15, 54E35

\footnotetext{
*Department of Mathematics, City College of New York, CUNY Email : rdkcc@ccny. cuny.edu

$\dagger^{\dagger}$ Department of Mathematics, Medgar Evers College, CUNY

Email : hpajoohesh@mec.cuny.edu

The second author wishes to acknowledge support for this research from the City University of New York (PSC-CUNY grant 68354-00 46).

Doi : 10.15672/HJMS.2016.394
} 


\section{Introduction}

Metrics have been generalized in many ways. Steve Matthews in [12] introduced partial metrics. His goal was to study the reality of finding closer and closer approximations to a given number (using, say, $n$-place decimal approximations), and showing that contractive algorithms would serve to find these approximations. Specifically, he identified the approximations as partially known points. For example an $n$-place decimal approximation to a number tells us in which interval of the form $\left[d, d+10^{-n}\right.$ ) the number lies; this interval has width $10^{-n}$; this tells the remaining uncertainty of the exact value of the point, and is usefully seen as its self-distance of this interval.

He then showed that the usual proof of the contraction fixed-point theorem worked in his more general spaces and the theorem in these spaces meant that contractive algorithms would converge to a fully known point - that is, a point whose distance to itself is 0 . His definition is:

1.1. Definition. A partial metric is a function $p: X \times X \rightarrow[0, \infty)$ satisfying the following conditions for every $x, y, z \in X$ :

$$
\begin{aligned}
& p(x, y) \geq p(x, x), \\
& p(x, y)=p(y, x), \\
& p(x, z)+p(y, y) \leq p(x, y)+p(y, z), \\
& x=y \text { if } p(x, y)=p(x, x)=p(y, y) .
\end{aligned}
$$

One can easily verify that a partial metric $p$ on the set $X$ is a metric if and only if $p(x, x)=0$ for every $x \in X$.

An example of a partial metric is $\vee:[0, \infty) \times[0, \infty) \rightarrow[0, \infty)$ such that $\vee(x, y)=$ $\max \{x, y\}$.

In [11] partial metrics were generalized by allowing $p: X \times X \rightarrow V$, where $V$ is a value quantale or a value lattice. In [10] completions of partial metric spaces were considered and it was shown that a new form of completion called the spherical completion is the same as the "Round ideal completion" which is important in computer science.

In [2], a relationship between partial metrics and metrics with a base point was discussed. In [6] partial metrics on $\ell$-groups (lattice ordered groups) were discussed. There it was shown that if $p_{n \vee}(x, y)=n(x \vee y)$ is a partial metric on an $\ell$-group $G$ then $n a+n b=n b+n a$ for all $a, b \in G$ (this property is sometimes called the commutativity of the $n$th power). So if $\mathcal{E}_{n}$ is the variety of $\ell$-groups so that $p_{n \vee}$ is a partial metric and $\mathcal{L}_{n}$ is the variety of $\ell$-groups such that the $n$th power commutes then $\mathcal{E}_{n} \subseteq \mathcal{L}_{n}$. There it was shown that if $n$ is prime then $\mathcal{E}_{n}=\mathcal{L}_{n}$.

Later in [4] it was shown that $\mathcal{E}_{n}=\mathcal{L}_{n}$ if and only if $n$ is prime. In [4] it was shown that $\mathcal{L}_{n} \cap \mathcal{A}^{2} \subseteq \mathcal{E}_{n}$ for every $n$, where $\mathcal{A}^{2}$ is the set of $\ell$-groups $G$ such that $G$ has an abelian convex normal $\ell$-subgroup $H$ such that $\frac{G}{H}$ is abelian. Further, if $n$ is prime then $\mathcal{L}_{n} \cap \mathcal{A}^{2}=\mathcal{E}_{n}$ but whether this equality holds for all $n$ was unanswered for some time. Then in [3] it was shown that this equality holds if $n=p q$ where $p$ and $q$ are two positive prime numbers. Later, in [13] it was shown that the equality holds for every $n$. These papers built another bridge between computer science and order theory. 
Metrics have also been generalized by allowing them to be valued in structures other than $\mathbb{R}$, such as abelian $\ell$-groups. When this is done and a notion of "positive" is given, many basic topology-like notions can be characterized with these structures - for example each Tychonoff topology ( [8]), each proximity, and each uniformity ( [7]) arises from a metric into such a structure with a subset of positives, and if symmetry is dropped then one obtains a quasimetric, and each topology ( [8]), each quasiproximity and each quasiuniformity ( [7]) as well as each neighborhood space, closure space and pretopology arises from a quasimetric into such a structure with a subset of positives (the last three are discussed in [9]). Similarly it was shown in [11] that each $T_{0}$ topology arises from a partial metric into such a space with a subset of positives.

When it comes to $\ell$-groups, a major tool that allows this generalization is that absolute value for abelian $\ell$-groups, like absolute value on the real line, yields a metric. More precisely the absolute value of the zero is zero, the absolute values of an element and that of its inverse are the same, and the triangle law holds. The triangle law fails for non-abelian $\ell$-groups; in fact in [5] it was shown that an $\ell$-group is abelian if and only if the triangle law for the absolute value holds. For a general $\ell$-group we only have that for each $x, y,|x+y| \leq 2(|x|+|y|)$. This led to defining $k$-metrics, see [14].

1.2. Definition. Let $k$ be a positive integer. A $k$-metric on a set $X$ is a function $d: X \times X \rightarrow \mathbb{R}$ such that for all $x, y, z \in X$ :

(pos) $d(x, y) \geq 0$

(id) $d(x, y)=0 \Leftrightarrow x=y$

(sym) $d(x, y)=d(y, x)$

(ktri) $d(x, y) \leq k(d(x, z)+d(z, y))$.

A $k$-metric space is a set $X \neq \emptyset$ with a $k$-metric on it.

If we allow $k$-metrics to be valued in a lattice ordered group $G$ rather than the reals, the absolute value of their difference, $d(x, y)=|x-y|$ will always be a 2-metric on G. (See [1] p. 296.)

Clearly if $d$ is a $k$-metric then it is $t$-metric for each integer $t \geq k$. Certainly every metric space is a 1-metric space and so it is a $k$-metric space whenever $1 \leq k \in \mathbb{N}$. But, the converse is not true; there are $k$-metric spaces which are not metric:

1.3. Example. Consider $d: \mathbb{R} \times \mathbb{R} \rightarrow \mathbb{R}$ defined by $d(x, y)=(x-y)^{2}$. Then $d$ is a 2-metric on $\mathbb{R}$ because for every two real numbers $a$ and $b$, we have $(a+b)^{2} \leq$ $2\left(a^{2}+b^{2}\right)$. But $d(x, y)=(x-y)^{2}$ is not a metric: for example $d(-1,1)=4 \not \leq$ $d(-1,0)+d(0,1)=1+1=2$. In general for every even integer $n, d(x, y)=|x-y|^{n}$ is a $2^{n}$-metric because for every $a, b \in \mathbb{R}$ we have $|a+b|^{n} \leq(|a|+|b|)^{n} \leq(2(|a| \mathrm{V}$ $|b|))^{n}=2^{n}(|a| \vee|b|)^{n} \leq 2^{n}\left(|a|^{n}+|b|^{n}\right)$. The last inequality holds since for every $a, b \in \mathbb{R}, a \vee b=a$ or $a \vee b=b$.

So $k$-metrics allow us to talk about distance between points and thus about sequences, series and convergence in a wider context. They relax triangularity and still the induced topology is metrizable, see [14]. Sometimes proving triangularity of metrics is challenging but proving triangularity for $k$-metrics can be much easier. As we see in this article many properties of metrics hold for $k$-metrics. 
The central point of this paper is to show that the basic theories of topology and uniformity are essentially the same for $k$-metrics as for metrics.

We recall that for $X \neq \emptyset$, a uniformity on $X$ is a set $\mathcal{U}$ of subsets of $X \times X$ such that:

(Id) $1_{X}=\bigcap \mathcal{U}$ where $1_{X}=\{(x, x): x \in X\}$, the identity map of $X$,

(Sym) if $U \in \mathcal{U}$ then $U^{-1} \in \mathcal{U}$ (where $U^{-1}=\{(y, x):(x, y) \in U\}$ )

(Int) if $U, V \in \mathcal{U}$ then $U \cap V \in \mathcal{U}$, and

(Com) if $U \in \mathcal{U}$ then $V \circ V \subseteq U$ for some $V \subseteq X \times X$, where $\circ$ denotes composition of relations.

Every uniformity $\mathcal{U}$ induces a topology, $\tau_{\mathcal{U}}$, whose open sets are those $T$ for which $x \in T \Rightarrow(\exists U \in \mathcal{U})(U[\{x\}] \subseteq T)$, where $U[\{x\}]=\{y \in X:(x, y) \in U\}$.

For a $k$-metric $d: X \times X \rightarrow \mathbb{R}$, if $x \in X$ and $r>0, N_{r}(x)=\{y: d(x, y) \leq r\}$ and $\tau_{d}=\left\{T \subseteq X:(\forall x \in T)(\exists r>0)\left(N_{r}(x) \subseteq T\right)\right\}$ is called the topology induced by the $k$-metric. Also, if $r>0, N_{r}=\{(x, y): x, y \in X \& d(x, y) \leq r\}$ and $\mathcal{U}_{d}=\{U \subseteq$ $\left.X \times X:(\exists r>0)\left(N_{r} \subseteq U\right)\right\}$ is the uniformity induced by the $k$-metric.

1.4. Lemma. For any $k$-metric space $(X, d), \tau_{d}$ is a topology, called the $k-$ metric topology on $X$ and $\mathfrak{U}_{d}$ is a uniformity, called the $k$-metric uniformity on $X$. Further, $\tau_{d}$ is the topology induced by $\mathcal{U}_{d}$.

Proof: That $\tau_{d}$ is a topology was shown in [14] (further, the reader can check it). Now we prove that $\mathcal{U}_{d}$ is a uniformity.

To show (Id) note that if $U \in \mathcal{U}_{d}$ then for some $r>0, N_{r} \subseteq U$; since for each $x \in X, d(x, x)=0 \leq r$, each $(x, x) \in N_{r}$, so $1_{X} \subseteq N_{r}$; thus $1_{X} \subseteq U$, and thus $1_{X} \subseteq \bigcap \mathcal{U}_{d}$; but if $(x, y) \notin 1_{X}$ then $x \neq y$ so $d(x, y) \neq 0$ so for some $r>0, d(x, y) \not \leq r$, so $(x, y) \notin N_{r}$, thus $(x, y) \notin \bigcap \mathcal{U}_{d}$, showing $\bigcap \mathcal{U}_{d} \subseteq 1_{X}$.

For (Sym), if $U \in \mathcal{U}$ then for some $r>0, N_{r} \subseteq U$, so $N_{r}=N_{r}^{-1} \subseteq U^{-1}$ thus $U^{-1} \in \mathcal{U}$.

For (Int), if $U, V \in \mathcal{U}_{d}$ then for some $r, s>0, N_{r} \subseteq U$ and $N_{s} \subseteq V$, thus $t=\min \{r, s\}>0$; also $N_{t} \subseteq N_{r} \cap N_{s} \subseteq U \cap V$, so $U \cap V \in \mathcal{U}_{d}$, and

For (Com) if $U \in \mathcal{U}$ then find $r>0$ such that $N_{r} \subseteq U$, and note that there is an $s>0$ so that $2 k s \leq r$. Let $V=N_{s} \in \mathcal{U}_{d}$ and if $(x, y),(y, z) \in N_{s}$ then $d(x, z) \leq k(d(x, y)+d(y, z)) \leq 2 k s \leq r$, so $N_{s} \circ N_{s} \subseteq N_{r} \subseteq U$.

Thus we can define uniform continuity, Cauchy sequences, continuity and limits for $k$-metrics exactly the way they are defined for metrics. Similarly we say a $k$ metric is complete if every Cauchy sequence converges.

\section{The contraction fixed-point theorem}

2.1. Definition. For a $k$-metric space $(X, d)$, a function $f: X \rightarrow X$ is a Lipschitz map with bound $q$ if $q$ is so that for each $x, y \in X, d(f(x), f(y)) \leq q d(x, y) ; f$ is a contraction if it is a Lipschitz map with bound $q<\frac{1}{k^{2}}$.

2.2. Lemma. If $f: X \rightarrow X$ is a Lipschitz map with bound $q$ on a $k$-metric space $(X, d)$, then $f$ is continuous.

Proof: To show continuity with respect to $\tau_{d}$, we establish that for each $r>0$ and $x \in X$, there is an $s>0$ such that $d(x, y) \leq s$ implies $d(f(x), f(y)) \leq r$. Let $s=\frac{r}{q}$; then if $d(x, y)<s$ we have $d(f(x), f(y))<q d(x, y)<q s=q\left(\frac{r}{q}\right)=r$. 
Note that the above in fact showed uniform continuity (in other words $x$ doesn't matter).

2.3. Lemma. If $f: X \rightarrow X$ is a Lipschitz map with bound $q$ on a k-metric space $(X, d)$, then for each $x \in X, n \in \mathbb{N} \cup\{0\}, d\left(x, f^{n}(x)\right) \leq\left(\sum_{i=1}^{n} k^{i} q^{i-1}\right) d(x, f(x))$.

Proof: For $n=0$ and all $x \in X$, this inequality says

$d\left(x, f^{0}(x)\right) \leq\left(\Sigma_{i=1}^{0} k^{i} q^{i-1}\right) d(x, f(x))$; that is, $d(x, x)=0 \leq(\Sigma \emptyset) d(x, f(x))$, which holds. Assume our inequality for $n$ and all $x \in X$. Then by the $k$-metric triangle inequality and the inductive hypothesis, $d\left(x, f^{n+1}(x)\right) \leq k(d(x, f(x))+$ $\left.d\left(f(x), f^{n}(f(x))\right)\right) \leq k d(x, f(x))+k d\left(f(x), f^{n}(f(x))\right) \leq$

$k d(x, f(x))+k\left(\sum_{i=1}^{n} k^{i} q^{i-1}\right) d(f(x), f(f(x))) \leq k d(x, f(x))+k\left(\sum_{i=1}^{n} k^{i} q^{i-1}\right) q d(x, f(x))=$ $k d(x, f(x))+\left(\sum_{i=1}^{n} k^{i+1} q^{i}\right) d(x, f(x))=k d(x, f(x))+\left(\sum_{i=2}^{n+1} k^{i} q^{i-1}\right) d(x, f(x))=$ $\left(\sum_{i=1}^{n+1} k^{i} q^{i-1}\right) d(x, f(x))$.

So our inequality holds for $n+1$ and arbitrary $x$, completing our inductive proof.

2.4. Theorem. Fixed point Theorem Let $(X, d)$ be a complete $k$-metric space. If $f: X \rightarrow X$ is a contraction then $f$ has a fixed point.

Proof: If $k=1$ then we have a metric and the result is true. Thus let $k>1$. Let $x_{0} \in X$ be any point and inductively define $x_{n}=f\left(x_{n-1}\right)$. Then for each $n, x_{n}=f^{n}\left(x_{0}\right)$. Now we show that the sequence $\left\{x_{n}\right\}$ is Cauchy: Let $\epsilon>0$; there is an $N$ such that $\frac{1}{k^{2 N-2}} \frac{1}{k-1} d\left(x_{0}, x_{1}\right)<\epsilon$. Now let $m \geq n \geq N$. Since $f$ is a Lipschitz map with bound $q<\frac{1}{k^{2}}$, by Lemma $2.3, d\left(x_{n}, x_{m}\right)=d\left(f^{n}\left(x_{0}\right), f^{m}\left(x_{0}\right)\right) \leq$ $q^{n}\left(d\left(x_{0}, f^{m-n}\left(x_{0}\right)\right)\right) \leq q^{n}\left(\sum_{i=1}^{m-n} k^{i} q^{i-1}\right) d\left(x_{0}, f\left(x_{0}\right)\right) \leq \frac{1}{k^{2 n}}\left(\sum_{i=1}^{m-n} \frac{1}{k^{i-2}}\right) d\left(x_{0}, x_{1}\right) \leq$ $\frac{1}{k^{2 n}}\left(\sum_{i=1}^{\infty} \frac{1}{k^{i-2}}\right) d\left(x_{0}, x_{1}\right)$

$=\frac{1}{k^{2 n-2}}\left(\sum_{i=1}^{\infty} \frac{1}{k^{2}}\right) d\left(x_{0}, x_{1}\right)=\frac{1}{k^{2 n-2}} \frac{1}{k-1} d\left(x_{0}, x_{1}\right) \leq \frac{1}{k^{2 N-2}} \frac{1}{k-1} d\left(x_{0}, x_{1}\right)<\epsilon$.

This proves that $\left\{x_{n}\right\}$ is a Cauchy sequence, and since $(X, d)$ is a complete $k$-metric space, it converges to a point, say $a$. Thus by the continuity of $f$ (shown in Lemma 2.2):

$f(a)=f\left(\lim _{n \rightarrow \infty} x_{n}\right)=\lim _{n \rightarrow \infty} f\left(x_{n}\right)=\lim _{n \rightarrow \infty} x_{n+1}=a$, so $a$ is a fixed point.

\section{Future Work}

The continuation of this research is considering $k$-partial metrics. We have a definition for $k$-partial metrics and we must verify that it follows the idea of partial metric and generalizes partial metrics in a way that keeps their properties and their relationship with other distance functions.

\section{References}

[1] Birkhoff, G., Lattice Theory, 3rd Edition, American Mathematical Society Colloquium Publications, Volume 25, 1967, Providence, RI.

[2] Bukatin, M., Kopperman, R., Matthews, S., and Pajoohesh, H., Partial Metric spaces, American Mathematical Monthly 116, 708-718, 2009.

[3] Darnel, M. and Holland, W.C., Minimal non-metabelian varieties of $\ell$-groups that contain no nonabelian o-groups, Communications in Algebra 42, 5100-5133, 2014. 
[4] Darnel, M., Holland, W.C., Pajoohesh, H.,The relationship of partial metric varieties and commuting powers varieties, Order, 30, no.2, 403-414, 2013.

[5] Holland, W. C., Intrinsic metrics for lattice-ordered groups, Algebra Universalis, 19, 142$150,1984$.

[6] Holland, W.C, Kopperman, R. and Pajoohesh, H., Intrinsic generalized metrics Algebra Universalis, 67, no.1, 1-18, 2012.

[7] Kopperman, R., Lengths on Semigroups and Groups, Semigroup Forum 25, 345-360, 1984.

[8] Kopperman, R., All Topologies Come From Generalized Metrics, Am. Math. Monthly 95, 89-97, 1988.

[9] Kopperman, R., Mynard, F., and Ruse, P., Quasi-metric representations of various categories of closure spaces, Topology Proceedings, 37: 331-347, 2011.

[10] Kopperman, R., Matthews, S., and Pajoohesh, H., Completions of partial metrics into value lattices, Topology and Applications 156, 1534-1544, 2009.

[11] Kopperman, R., Matthews, S., and Pajoohesh, H., Universal partial metrizability, Applied General Topology 5, 115-127 2004.

[12] Matthews, S. G., "Partial metric topology", Proc. 8th summer conference on topology and its applications, ed S. Andima et al., New York Academy of Sciences Annals, 728, 183-197, 1994.

[13] Pajoohesh, H., The relationship of partial metric varieties and commuting powers varieties II, Algebra Universalis, 73, issue 3-4, 291-295, 2015.

[14] Pajoohesh, H., k-metric spaces, Algebra Universalis, 69, no.1, 27-43, 2013. 